\title{
A Case of Elephantiasis Nostras Verrucosa
}

\author{
Yun-Seok Yang, M.D., Jae-Jun Ahn, M.D., Sik Haw, M.D., Min-Kyung Shin, M.D., \\ Choong-Rim Haw, M.D.
}

Department of Dermatology, School of Medicine, Kyunghee University, Seoul, Korea

Elephantiasis nostras verrucosa (ENV) is a rare clinical condition associated with chronic non-filarial lymphedema caused by bacterial or non-infectious lymphatic obstruction. A variety of etiologies, including infection, tumor obstruction, trauma, radiation, chronic venous stasis, congestive heart failure, and obesity, can lead to chronic lymphatic obstruction and edema. Mossy papules, plaques, and cobblestone-like nodules are clinically impressive features of ENV, but biopsy reveals only moderately abnormal findings such as pseudoepitheliomatous hyperplasia, dilated lymphatic spaces, fibrous tissue hyperplasia, and chronic inflammation. We present a case of ENV in a 67-year-old man with a 10-year history of multiple nodules and verrucous plaques on both feet. Microbiology ruled out a filarial infection. Nodule biopsy revealed pseudoepitheliomatous hyperplasia, marked dermal fibrosis, and a chronic inflammatory infiltrate. No evidence of carcinoma was identified. Both venous stasis and recurrent cellulitis could contribute to the dermal fibrotic changes of the lesions. However, before the recurrent cellulitis, he did not have any nodular lesions on his feet despite a 10-year history of venous disease. Therefore, this case suggests that venous stasis alone cannot produce the fibrotic nodular lesions of ENV. (Ann Dermatol 21(3) 326 329, 2009)

\section{-Keywords-}

Cellulitis, Elephantiasis, Elephantiasis nostras verrucosa, Venous stasis

\section{Received March 13, 2009, Accepted for publication April 2, 2009}

Reprint request to: Choong-Rim Haw, M.D., Department of Dermatology, School of Medicine, Kyunghee University, 1, Hoegi-dong, Dongdaemun-gu, Seoul 130-702, Korea. Tel: 82-2-958-8510, Fax: 82-2-969-6538, E-mail: crhaw@khmc.or.kr

\section{INTRODUCTION}

In 1969, Castellani ${ }^{1}$ classified elephantiasis into four subtypes: 1) elephantiasis tropica - due to filariasis; 2) elephantiasis nostras - due to bacterial infection; 3) elephantiasis symptomatica - due to mycotic, syphilitic, tuberculoid, neoplastic, or traumatic causes of lymphatic obstruction; and 4) elephantiasis congenita - associated with inherited disorders such as Milroy's disease. Elephantiasis nostras verrucosa (ENV) was originally defined as a condition resulting from lymphatic blockage caused by recurrent bacterial infection and also called lymphangitis recurrens elephantogenica. However, many recent papers have included neoplastic, traumatic, and other noninfectious causes of lymphatic obstruction in the definition of $\mathrm{ENV}^{2-4}$.

ENV is a rare disease, and only one case of elephantiasis nostras has been described in the Korean dermatologic literature $^{5}$. Herein, we report a case of ENV due to recurrent cellulitis and chronic venous stasis and offer suggestions concerning its possible pathogenesis.

\section{CASE REPORT}

A 67-year-old man presented with a chief complaint of slowly enlarging multiple hard papules and plaques on both feet over the past 10 years. Ten years ago, he had been in the habit of drinking raw deer blood as a health food, and after that suffered from toxic erythema with some erosion on both feet. Subsequently, he was hospitalized several times for recurrent cellulitis of both feet. He first noticed venous symptoms such as heaviness of the legs, swelling, and mild pain while standing 20 years prior to presentation at our hospital but had declined any interventions that were recommended at other hospitals. Examination of the feet revealed confluent pinkish or brownish verrucous papules and plaques with a mosscovered appearance surrounding the peripheries of both 

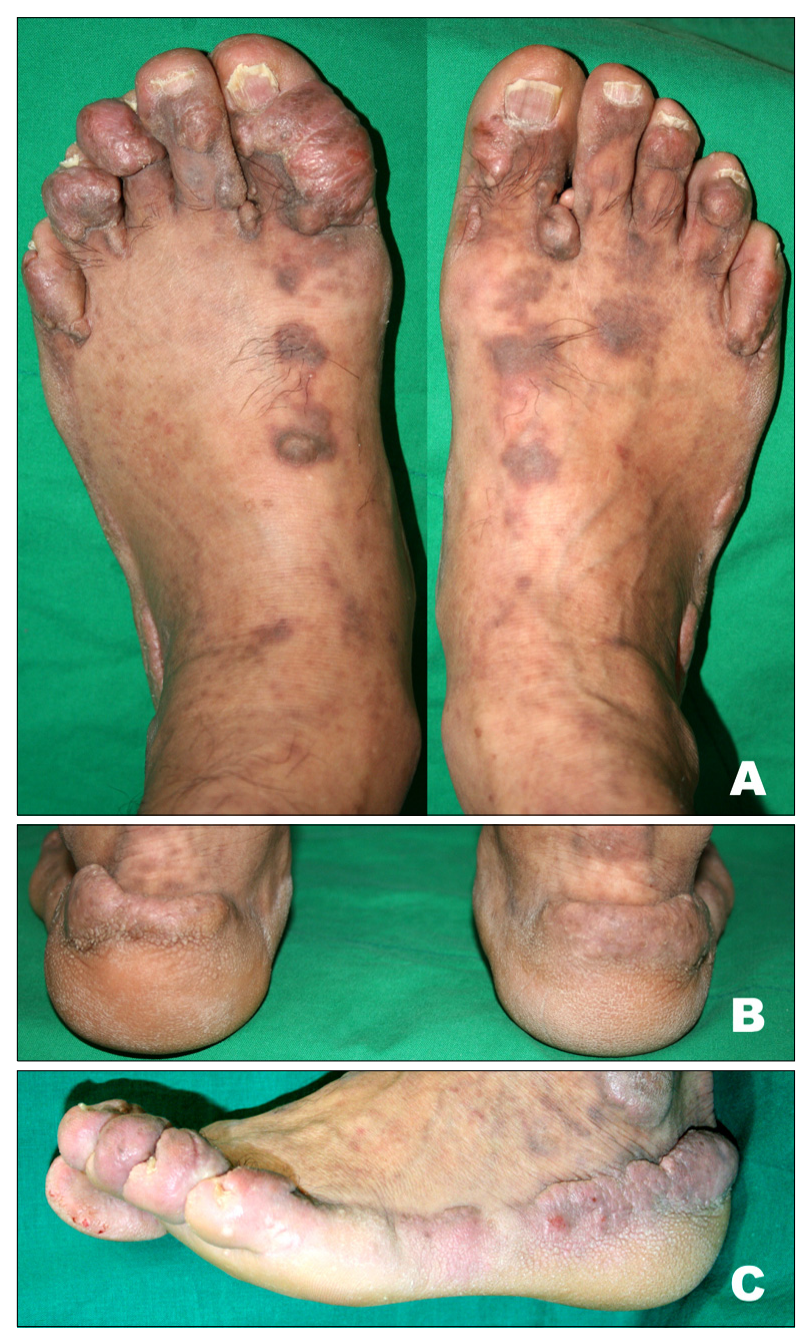

Fig. 1. (A) Pink or brown hard papules and nodules on both toes and toe webs. $(B, C)$ Plaques with a moss-covered appearance surround the heel and outer edge of both feet. feet from the tip of the toe to the back of the heel bilaterally (Fig. 1). Both of his legs exhibited brownish, non-pruritic maculopatches that had been present for 10 years after the toxic erythema, varicose veins, and mild, nonpitting edema below the knee. The patient reported no history of trauma, surgery or radiation, and no travel outside of Korea. He had no family history of familial lymphedema. Laboratory investigations showed a mildly elevated ESR $(37 \mathrm{~mm} / \mathrm{hr}$ ), but a complete blood count with differential, biochemical and serologic tests were normal except for a glucose of $193 \mathrm{mg} / \mathrm{dl}$ due to uncontrolled type 2 diabetes mellitus. A peripheral blood smear and specific antigen testing for microfilaria resulted in negative findings. Microscopic examination of toe web scrapings on the $\mathrm{KOH}$ preparation and fungal culture were negative. Lower extremity computed tomographic angiography (CTA) revealed varicose veins with early venous drainage at both feet and ankles, and suggested a hyperemic state due to chronic inflammation. Excisional biopsy was performed on the great toe, with results showing hyperkeratosis and pseudoepitheliomatous hyperplasia as well as a fibrotic dermis with some dilated lymphatic channels (Fig. 2). Periodic acid-Schiff, and acidfast bacilli stains were negative for fungal and mycobacterial organisms. Thus, a diagnosis of ENV was made based on these findings. The patient was treated with oral etretinate at a dose of $0.5 \mathrm{mg} / \mathrm{kg} /$ day and topical salicylic acid, but showed little improvement after 3 months. He was referred to the department of general surgery for the venous problem but refused any interventions. Unfortunately, the patient was lost to follow-up after 3 months of treatment.
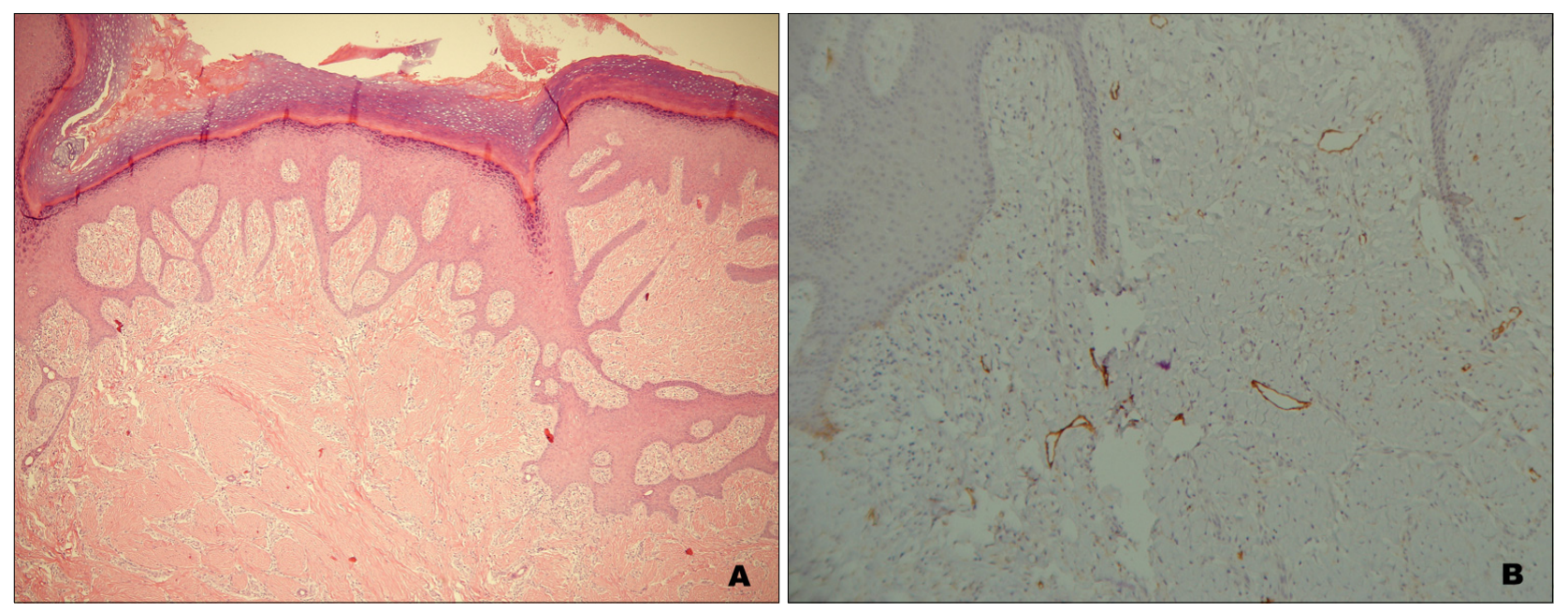

Fig. 2. (A) Histologic examination demonstrates hyperkeratosis, papillomatosis, and pseudoepitheliomatous hyperplasia of the epidermis and marked dermal fibrosis $(\mathrm{H} \& \mathrm{E}, \times 40)$. (B) Dilated lymphatic channels are observed in the upper dermis $(\mathrm{D} 2-40, \times 100)$. 


\section{DISCUSSION}

Classically, the term elephantiasis applies to swelling of the legs caused by helmintic Wuchereria species. In such cases, patients are infested by filarial worms in tropical areas, where such parasites are endemic. The worms block lymphatic channels, leading to severe chronic swelling in the lower legs and genitals. In 1934, the term "nostras" was added to distinguish lymphedematous disorders of temperate regions not caused by filariasis ${ }^{6}$. In recent papers, however, the term ENV is more often used to denote a dermatological complication of bacterial or non-infectious lymphatic obstruction characterized by dermal fibrosis and epidermal changes consisting of hyperkeratotic, verrucous, and papillomatous lesions ${ }^{2,4,7,8}$. The lower extremities are the most common site of ENV, but any area with chronic lymphedema can be affected ${ }^{2}$.

Although recurrent streptococcal lymphangitis may contribute to the origin of ENV, the exact pathogenesis of the disorder is not yet clear ${ }^{4}$. A variety of etiologies can lead to lymphatic obstruction and edema, and the resultant prolonged accumulation of protein-rich interstitial fluid in the affected area induces fibroblast proliferation and increases susceptibility to infection and inflammation, which in turn leads to further fibrosis of the dermis and lymph channels ${ }^{2,4,9}$. The underlying causes of lymphatic obstruction can be bacterial infection, malignancy, lymphangioma, prior surgery or trauma, and lymphatic fibrosis due to radiation therapy, although chronic venous stasis, obesity, and scleroderma should also be considered ${ }^{4,7}$. In chronic venous disease, activated leukocytes may migrate out of the vasculature and release TGF- $\beta_{1}$, stimulating collagen production by dermal fibroblasts, which culminates in dermal and lymphatic fibrosis ${ }^{10}$. In our case, the patient had a history of recurrent cellulitis and also had chronic venous stasis, both of which could contribute to the dermal fibrotic changes. However, before the recurrent cellulitis, he did not have any nodular lesions on his feet despite suffering from venous disease for the previous 10 years, suggesting that venous stasis alone did not cause the fibrotic nodular lesions. This agrees with a previous report that mere lymphatic obstruction or venous stasis does not cause dermal fibrosis without recurrent bacterial lymphangitis ${ }^{11}$.

Histologically, pseudoepitheliomatous hyperplasia, dilated lymphatic spaces, and fibrosis are characteristic of ENV, but not all of these findings may be present during the advanced stage ${ }^{2}$. The patient's history and characteristic skin changes were sufficient to diagnose ENV. However, histopathologic examination of biopsy specimens and imaging techniques such as lymphangiography, lymphoscintigraphy, computed tomography (CT), and magnetic resonance imaging (MRI) may provide further cues to differentiate ENV from other disorders such as an obstructing tumor.

Differential diagnoses include chromoblastomycosis, filariasis, lipodermatosclerosis, verrucous carcinoma, papular mucinosis, and pretibial myxedema ${ }^{2}$. Chromoblastomycosis or filariasis can be diagnosed by laboratory identification of causative organisms. Lipodermatosclerosis is a condition that affects the skin just above the ankle in patients with long-standing venous disease, resulting in chronic venous insufficiency, with biopsy revealing fibrin deposits around the capillaries ${ }^{12}$. Verrucous carcinoma and papular mucinosis can be ruled out by histopathologic findings such as atypical keratinocytes in the verrucous carcinoma or acid glycosaminoglycan deposition in the papular mucinosis. Pretibial myxedema is a manifestation of Graves' disease preceded by ophthalmopathy and thyroid gland enlargement, and is histologically differentiated from ENV by the presence of increased hyaluronan and chondroitin sulfates in the dermis, but ENV can be a rare variant of pretibial myxedema $^{13}$. ENV may also occur as a sequela of chronic venous stasis as mentioned above. However, ENV should be differentiated from venous stasis dermatitis, which typically leads to pitting edema associated with erythematous, scaly, pruritic patches ${ }^{9}$. Our patient did not complain of any pruritus or scales, and his lower legs showed non-pitting edema. Thus, the lesions on his feet cannot be diagnosed as venous stasis dermatitis.

Treatments for ENV include conservative measures to reduce lymphostasis (compressive stockings, mechanical massage), medical management of edema by diuretics, and prevention of recurrent infection by prolonged courses of antibiotics ${ }^{2}$. Oral retinoids may be effective, but the sustainability of these results appears variable ${ }^{14}$. Rarely, surgical debridement or amputation is performed in recalcitrant cases ${ }^{15}$.

Chronic nodular skin changes are almost always progressive, and management of advanced stages usually results in unsatisfactory outcomes. Therefore, it is important to recognize this rare condition in its initial stages and control infection and edema as soon as possible to prevent debilitating deformities. In addition, the underlying cause of lymphatic obstruction should be investigated and treated to the maximal extent possible.

\section{REFERENCES}

1. Castellani A. Researches on elephantiasis nostras and elephantiasis tropica with special regard to their initial stage 
of recurring lymphangitis (lymphangitis recurrens elephantogenica). J Trop Med Hyg 1969;72:89-97.

2. Sisto K, Khachemoune A. Elephantiasis nostras verrucosa: a review. Am J Clin Dermatol 2008;9:141-146.

3. Routh HB. Elephantiasis. Int J Dermatol 1992;31:845-852.

4. Schissel DJ, Hivnor C, Elston DM. Elephantiasis nostras verrucosa. Cutis 1998;62:77-80.

5. Choi JM, Park CJ, Yi JY. A case of elephantiasis nostras. Korean J Dermatol 1998;36:1098-1100.

6. Castellani A. Elephantiasis nostras. J Trop Med Hyg 1934;37: 257-264.

7. Duckworth AL, Husain J, Deheer P. Elephantiasis nostras verrucosa or "mossy foot lesions" in lymphedema praecox: report of a case. J Am Podiatr Med Assoc 2008;98:66-69.

8. Turhan E, Ege A, Keser S, Bayar A. Elephantiasis nostras verrucosa complicated with chronic tibial osteomyelitis. Arch Orthop Trauma Surg 2008;128:1183-1186.

9. Boyd J, Sloan S, Meffert J. Elephantiasis nostrum verrucosa of the abdomen: clinical results with tazarotene. J Drugs Der- matol 2004;3:446-448.

10. Bergan JJ, Schmid-Schonbein GW, Smith PD, Nicolaides AN, Boisseau MR, Eklof B. Chronic venous disease. N Engl J Med 2006;355:488-498.

11. Chernosky ME, Derbes VJ. Elephantiasis nostras of the abdominal wall. Arch Dermatol 1966;94:757-762.

12. Barron GS, Jacob SE, Kirsner RS. Dermatologic complications of chronic venous disease: medical management and beyond. Ann Vasc Surg 2007;21:652-662.

13. Kakati S, Doley B, Pal S, Deka UJ. Elephantiasis Nostras Verrucosa: a rare thyroid dermopathy in Graves' disease. J Assoc Physicians India 2005;53:571-572.

14. Zouboulis CC, Biczo S, Gollnick H, Reupke HJ, Rinck G, Szabo M, et al. Elephantiasis nostras verrucosa: beneficial effect of oral etretinate therapy. Br J Dermatol 1992;127: 411-416.

15. Iwao F, Sato-Matsumura KC, Sawamura D, Shimizu $H$. Elephantiasis nostras verrucosa successfully treated by surgical debridement. Dermatol Surg 2004;30:939-941. 